\title{
New insights into the problem with a singular drift term in the complex Langevin method
}

\author{
Jun Nishimura, ${ }^{1,2}$ and Shinji Shimasaki周 \\ ${ }^{1}$ KEK Theory Center, \\ High Energy Accelerator Research Organization, \\ Tsukuba 305-0801, Japan \\ ${ }^{2}$ Department of Particle and Nuclear Physics, \\ School of High Energy Accelerator Science, \\ Graduate University for Advanced Studies (SOKENDAI), \\ Tsukuba 305-0801, Japan
}

(Dated: April, 2015; preprint: KEK-TH-1816)

\begin{abstract}
The complex Langevin method aims at performing path integral with a complex action numerically based on complexification of the original real dynamical variables. One of the poorly understood issues concerns occasional failure in the presence of logarithmic singularities in the action, which appear, for instance, from the fermion determinant in finite density QCD. We point out that the failure should be attributed to the breakdown of the relation between the complex weight that satisfies the Fokker-Planck equation and the probability distribution associated with the stochastic process. In fact, this problem can occur, in general, when the stochastic process involves a singular drift term. We show, however, in a simple example that there exists a parameter region in which the method works, although the standard reweighting method is hardly applicable.

PACS numbers: 05.10.Ln, 02.70.Tt, 12.38.Gc
\end{abstract}

\section{INTRODUCTION}

The path integral formulation plays an important role in nonperturbative studies of quantum field theories due to the possibility of using Monte Carlo methods. The basic idea is to generate field configurations with a probability $\mathrm{e}^{-S}$ and to evaluate the path integral by taking a statistical average. It is not straightforward, however, to apply such an approach to cases with a complex action $S$ since one can no longer view $\mathrm{e}^{-S}$ as the probability distribution. This "complex action problem" occurs, for instance, in QCD at finite density or with a theta term, in Chern-Simons gauge theories, and in chiral gauge theories. It also appears in supersymmetric gauge theories and matrix models, which are relevant in nonperturbative studies of superstring theory.

The complex Langevin method (CLM) [1, 2] attempts to solve this problem by extending the idea of stochastic quantization [3] for ordinary systems with a real action to the case with a complex action. This necessarily requires complexifying the real dynamical variables that appear in the original path integral. A stochastic process for the complexified variables is defined by the Langevin equation with the complex action, and expectation values in the original path integral are calculated from an average of corresponding quantities over the stochastic process. See Ref. [4] for a pedagogical review on this method.

One of the recent developments in this method is the

*Electronic address: jnishi@post.kek.jp

${ }^{\dagger}$ Electronic address: simasaki@post.kek.jp clarification of a necessary condition for convergence to correct results [5]. In fact, there is a lot of freedom in defining the stochastic process that corresponds formally to the original path integral. By using this freedom, one can try to satisfy the conditions for convergence. The socalled gauge cooling [6] is a new technique of this kind, which has made it possible to study finite density QCD in the high temperature (deconfined) phase [7].

Despite these developments, there is still a puzzle concerning the treatment of logarithmic singularities in the action [8-10]. For instance, the effective action for QCD involves the logarithm of a determinant, which represents the effects of dynamical quarks. At finite density, the determinant $\Delta$ becomes complex in general, which causes the complex action problem. In this case, the effective action has an ambiguity due to the branch cut of the logarithm; the phase of the determinant can be defined only modulo $2 \pi$. For instance, one may use the drift term $\partial \log \Delta=\Delta^{-1} \partial \Delta$ in the complex Langevin equation, which corresponds to regarding the $-\log \Delta$ term in the action as a multi-valued function. It has been found, however, that the CLM with this prescription can give wrong results in a simplified model when the phase of the determinant rotates frequently during the stochastic process [8]. The wrong results turn out to be close to (but not equal to) the results obtained for the phasequenched model, in which the phase of the determinant is quenched. This problem has not shown up yet in recent QCD simulations at finite density [11, 12].

Here we provide new insights into this issue from the viewpoint of the Fokker-Planck (FP) equation. We start with simple examples and discuss more general cases towards the end. 


\section{ONE-VARIABLE CASE}

\section{A. Formulation of the CLM}

Let us consider a simple example defined by the partition function

$$
Z=\int d x w(x), \quad w(x)=(x+i \alpha)^{p} \mathrm{e}^{-x^{2} / 2},
$$

where $x$ is a real variable and $\alpha$ and $p$ are real parameters. For $\alpha \neq 0$ and $p \neq 0$, the weight $w(x)$ is complex, and the idea of important sampling cannot be applied to (1) by regarding $w(x)$ as the Boltzmann weight.

Following the usual procedure in CLM, we define the drift term

$$
v(x)=w(x)^{-1} \frac{\partial w(x)}{\partial x}=\frac{p}{x+i \alpha}-x,
$$

and complexify the variable as $x \mapsto z=x+i y$. The action

$$
S(z)=-\log w(z)=-p \log (z+i \alpha)+z^{2} / 2
$$

after the complexification has a logarithmic singularity at $z=-i \alpha$ for $p \neq 0$, which causes the aforementioned ambiguity due to the branch cut. However, we emphasize that this is not an issue, in general, because all we need in formulating the CLM, as we show below, is the single-valuedness of the drift term $v(z)$ after the complexification and the single-valuedness of the complex weight $w(x)$ as a function of $x$. These are satisfied in the present case even for a non-integer $p$.

The complex Langevin equation corresponding to the partition function (11) can be written as

$$
\frac{d z}{d t}=v(z)+\eta(t)=\frac{p}{z+i \alpha}-z+\eta(t),
$$

where $\eta(t)$ represents a real Gaussian noise satisfying $\left\langle\eta(t) \eta\left(t^{\prime}\right)\right\rangle=2 \delta\left(t-t^{\prime}\right)$. We define the probability distribution $P(x, y ; t)$ of $x(t)$ and $y(t)$ at the Langevin time $t$. Its time evolution follows the FP-like equation

$$
\begin{aligned}
\frac{\partial}{\partial t} P= & L P \\
= & \frac{\partial}{\partial x}\left\{-(\operatorname{Re} v)_{z=x+i y}+\frac{\partial}{\partial x}\right\} P \\
& +\frac{\partial}{\partial y}\left\{-(\operatorname{Im} v)_{z=x+i y} P\right\} .
\end{aligned}
$$

The crucial point in the CLM is that there exists a complex weight $\rho(x ; t)$, which is related to the probability distribution $P(x, y ; t)$ through

$$
\int \mathcal{O}(x) \rho(x ; t) d x=\int \mathcal{O}(x+i y) P(x, y ; t) d x d y
$$

under certain conditions [5], where $\mathcal{O}(x)$ are observables that admit holomorphic extension to $\mathcal{O}(x+i y)$. The evolution of $\rho$ follows the usual FP equation

$$
\begin{aligned}
\frac{\partial}{\partial t} \rho & =L_{0} \rho, \\
L_{0} & =\frac{\partial}{\partial x}\left(-v(x)+\frac{\partial}{\partial x}\right),
\end{aligned}
$$

which has a time-independent solution $\rho(x ; t) \propto w(x)$, with $w(x)$ given in (11) since it is annihilated by the operator in parenthesis in (9). Thus, the necessary and sufficient conditions for being able to calculate the expectation value of $\mathcal{O}$ with respect to (1) by the CLM are:

i) The relation (7) between $\rho$ and $P$ holds.

ii) The solution $\rho(x ; t)$ of the FP equation (8) asymptotes to $w(x)$ as $t \rightarrow \infty$ up to some constant factor.

As possible observables in the present example (11), we consider $\mathcal{O}=x^{k}$, where $k$ is a positive integer. Assuming the ergodicity of the stochastic process, the right-hand side of (7) at $t=\infty$ can be evaluated by taking the time average of $z(t)^{k}$, where $z(t)$ is obtained by solving (4). We find numerically that this method gives correct results only for sufficiently large $|\alpha|$ for each $p$. In what follows, we clarify the reason why it fails at small $|\alpha|$.

\section{B. Spectrum of the "FP Hamiltonian"}

First we have investigated numerically the eigenvalue spectrum of the "FP Hamiltonian" $\left(-L_{0}\right)$ defined by (9), assuming that the complex weight $\rho(x)$ falls off rapidly as $|x| \rightarrow \infty$.

As is clear from what we wrote above, we have an eigenfunction $\rho(x)=w(x)$ with zero eigenvalue for arbitrary $p$ and $\alpha$. When $p$ is a positive odd integer and $\alpha=0$, we have another zero mode $\rho(x)=|x|^{p} \mathrm{e}^{-x^{2} / 2}$. For $p>1$, negative eigenvalues appear in the small $|\alpha|$ region. (Note that, when $\alpha=0$, we have an eigenfunction $\rho(x)=x \mathrm{e}^{-x^{2} / 2}$, which corresponds to the smallest eigenvalue $\lambda=-(p-1)$ for any $p$.) Thus, we find that the desired solution $\rho(x ; t) \propto w(x)$ is obtained in the longtime limit of the FP equation (8) at arbitrary $\alpha$ for $p<1$ and at sufficiently large $|\alpha|$ for $p>1$.

In the parameter region where we have negative modes, the complex weight diverges as $\rho(x ; t) \propto e^{\left|\lambda_{\min }\right| t} \rho_{\min }(x)$, where $\lambda_{\min }$ is the smallest eigenvalue and $\rho_{\min }(x)$ is the corresponding eigenfunction. Clearly this behavior is incompatible with the relation (7) considering that $P(x, y ; t) \geq 0$ and $\int d x d y P(x, y ; t)=1$. This implies that the relation (7) between $\rho$ and $P$ must be broken at least in this region.

Applying this kind of argument to a general multivariable case, we find that the condition like ii) in section II A is automatically satisfied if the condition like i) holds, as far as the probability distribution $P$ asymptotes to a unique function. 


\section{The relation between $\rho$ and $P$}

Let us then consider what can go wrong with (7). In the derivation of (7) given in Ref. [5], the authors use

$$
\begin{aligned}
& \int \mathcal{O}(x+i y) P(x, y ; t) d x d y \\
= & \int \mathcal{O}(x+i y ; t) P(x, y ; 0) d x d y,
\end{aligned}
$$

where $\mathcal{O}(x+i y ; t)$ is defined by solving

$$
\frac{d}{d t} \mathcal{O}(x+i y ; t)=L^{\top} \mathcal{O}(x+i y ; t)
$$

with the initial condition $\mathcal{O}(x+i y ; 0)=\mathcal{O}(x+i y)$. The symbol $L^{\top}$ represents an operator satisfying $\left\langle L^{\top} f, g\right\rangle=$ $\langle f, L g\rangle$, where $\langle f, g\rangle \equiv \int f(x, y) g(x, y) d x d y$, assuming that $f$ and $g$ are regular functions with sufficiently fast fall-off as $|x|,|y| \rightarrow \infty$.

In order to prove (10), they consider [5]

$$
F(\tau)=\int \mathcal{O}(x+i y ; \tau) P(x, y ; t-\tau) d x d y
$$

for $0 \leq \tau \leq t$, which interpolates both sides of Eq. (10). Taking the derivative with respect to $\tau$, they get

$$
\begin{aligned}
\frac{d}{d \tau} F(\tau)= & \int\left\{L^{\top} \mathcal{O}(x+i y ; \tau)\right\} P(x, y ; t-\tau) d x d y \\
& -\int \mathcal{O}(x+i y ; \tau) L P(x, y ; t-\tau) d x d y
\end{aligned}
$$

Naively, the two terms cancel through integrating by parts, which implies that $F(\tau)$ is independent of $\tau$ and hence (10). In order to justify the partial integration, however, one should be able to neglect the boundary terms. This requires that $P(x, y ; t)$ decreases sufficiently fast as $|x|,|y| \rightarrow \infty[5]$. In addition to this requirement, one also needs the holomorphy of the drift and of the observables to prove (7).

\section{Diverging boundary terms due to singularity}

In the present example, the fast fall-off of $P(x, y ; t)$ as $|x|,|y| \rightarrow \infty$ is satisfied due to the $-z$ term in (4). However, we should be careful of the singularity at $(x, y)=(0,-\alpha)$. In order for the boundary terms to be neglected, it is required that the limits

$$
\begin{array}{r}
\lim _{x \rightarrow 0}\left[x \int \frac{\mathcal{O}(z ; \tau)}{|z+i \alpha|^{2}} P(x, y ; t-\tau) d y\right], \\
\lim _{y \rightarrow-\alpha}\left[(y+\alpha) \int \frac{\mathcal{O}(z ; \tau)}{|z+i \alpha|^{2}} P(x, y ; t-\tau) d x\right]
\end{array}
$$

should exist for arbitrary $t$ and $\tau$. Note, in particular, that $\mathcal{O}(z ; \tau)$ obtained by solving (11) is highly singular at $z=-i \alpha$ since the operator $L$ appearing on the righthand side of (11) involves the singularity. For instance, let us take the $n$th derivative of the above expressions with respect to $\tau$ at $\tau=0$. Using (11), we obtain terms such as

$$
\lim _{x \rightarrow 0}\left[x \int \frac{\left(L^{\top}\right)^{n} \mathcal{O}(z)}{|z+i \alpha|^{2}} P(x, y ; t) d y\right]
$$

which diverges as $\sim \frac{1}{|x|^{(2 n-1)}}$ for $n \geq 1$ if $P(x, y ; t)$ is nonzero at $(x, y)=(0,-\alpha)$.

In order to describe the actual situation, let us define the radial distribution

$$
\varphi(r)=\frac{1}{2 \pi r} \int P(x, y, \infty) \delta\left(\sqrt{x^{2}+(y+\alpha)^{2}}-r\right) d x d y
$$

around the singular point $(x, y)=(0,-\alpha)$. For small $|\alpha|$, we observe that $\varphi(r) \sim r$ at small $r$ as long as $p$ is not very large. In this case, (16) still diverges for sufficiently large $n$, and the relation (7) between $\rho$ and $P$ can be violated. Indeed we find that the CLM yields wrong results in such a parameter region.

\section{E. Results for large $p$}

In the partition function (1), it is the prefactor $(x+i \alpha)^{p}$ that causes the complex action problem. In view of this, one might think that the CLM fails when the phase of $(z(t)+i \alpha)^{p}$ rotates frequently during the time evolution from (4). We find that this is not necessarily the case.

In order to demonstrate this point, we present our results for large $p$. Figure 1 shows that the CLM reproduces the exact results for $|\alpha| \gtrsim 14$ at $p=50$. From Fig. 2, we find for $\alpha=14$ that $\varphi(r)=0$ at $r \lesssim 6$, although we observe that the phase of $(z(t)+i \alpha)^{50}$ rotates frequently during the stochastic process.

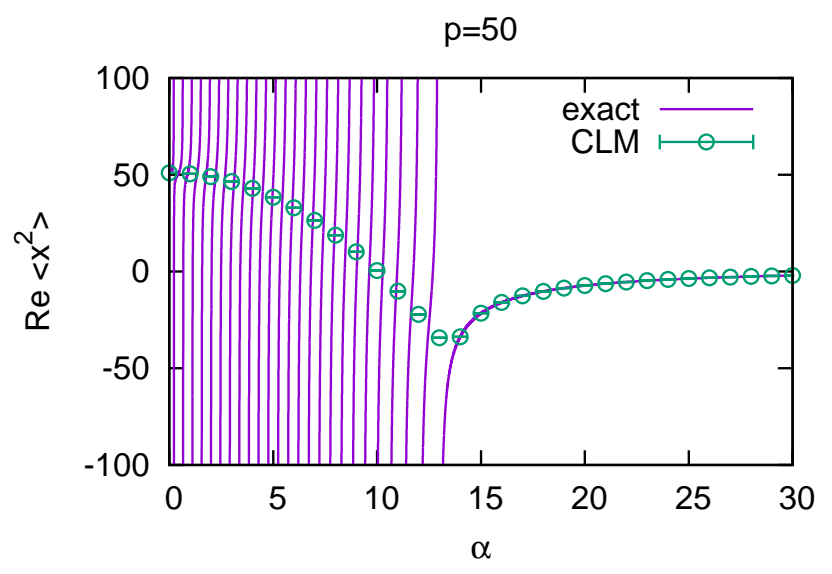

FIG. 1: The real part of the expectation value $\left\langle x^{2}\right\rangle$ obtained by the CLM (4) is plotted against $\alpha$ for $p=50$. The solid line represents the exact result obtained analytically by the Gaussian integration. 


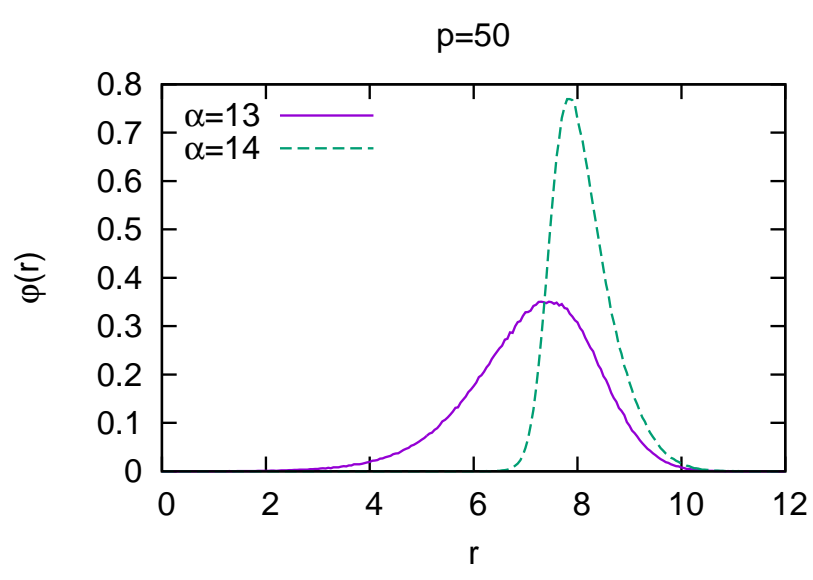

FIG. 2: The radial distribution $\varphi(r)$ defined in (17) is plotted for $p=50$ with $\alpha=13$ (solid line) and $\alpha=14$ (dashed line).

We would also like to mention that the complex action problem is extremely severe at $\alpha=14$ and $p=50$. As a standard quantity that measures the severeness of the complex action problem, let us consider

$$
R=\left\langle\frac{w(x)}{|w(x)|}\right\rangle_{0}=\left\langle\frac{(x+i \alpha)^{p}}{|x+i \alpha|^{p}}\right\rangle_{0}=\frac{Z}{Z_{0}},
$$

where $Z_{0}$ is the partition function of the phase-quenched model $Z_{0}=\int d x|w(x)|$ and the expectation value $\langle\cdot\rangle_{0}$ is taken with respect to it. In the present case, both $Z$ and $Z_{0}$ can be calculated analytically by performing the Gaussian integration. We find that $R \sim-7.4 \times 10^{-5}$ at $\alpha=14$ and $p=50$. One can imagine how hard it is to obtain correct results if one performs a Monte Carlo simulation of the phase-quenched model and applies the standard reweighting formula to obtain the expectation values with respect to the original partition function (11). Thus, the advantage of the CLM over the reweighting method can be appreciated even in this simple one-variable case.

\section{F. Non-logarithmic singularities}

Another interesting implication of our argument is that the possible failure of the CLM is not specific to logarithmic singularities in the action. Indeed we have found that the CLM can fail for the weight $w(x)=e^{-S(x)}$ with the action

$$
S(x)=\beta(x+i \alpha)^{-2}+x^{2} / 2 .
$$

Note that the action $S(z)$ after complexification does not involve a logarithmic singularity, which means, in particular, that there is no issue of ambiguity associated with the branch cut. Nevertheless, we find that the CLM fails at $|\alpha| \lesssim 1.2$ for $\beta=1$ and at $|\alpha| \lesssim 1.7$ for $\beta=-1$. On the other hand, from the studies of the eigenvalue spectrum of $\left(-L_{0}\right)$, we find that $\rho(x ; t) \propto e^{-S(x)}$ is obtained in the long-time limit of the FP equation (8) for arbitrary $\alpha$ with $\beta= \pm 1$. Therefore, the failure of the CLM at small $|\alpha|$ should be attributed to the violation of (7) due to the singularity in the drift term $v(z)=2 \beta(z+i \alpha)^{-3}-z$. This is also confirmed from the behavior of the radial distribution (17).

\section{TWO-VARIABLE CASE}

Our argument applies also to the case with multiple variables. To make this clear, let us consider a case with two variables given by

$$
\begin{aligned}
Z & =\int d x_{1} d x_{2} w\left(x_{1}, x_{2}\right), \\
w\left(x_{1}, x_{2}\right) & =\left(x_{1}+i x_{2}\right)^{p} \mathrm{e}^{-\left(x_{1}\right)^{2} / 2-\left(x_{2}-\alpha\right)^{2} / 2},
\end{aligned}
$$

where $x_{1}$ and $x_{2}$ are real variables. The parameter $\alpha$ is real, while $p$ is a positive integer.

We have studied numerically the eigenvalue spectrum of the operator $\left(-L_{0}\right)$, assuming that the complex weight $\rho\left(x_{1}, x_{2}\right)$ falls off rapidly as $\left(x_{1}\right)^{2}+\left(x_{2}\right)^{2} \rightarrow \infty$. First we obtain the desired zero mode $\rho\left(x_{1}, x_{2}\right)=w\left(x_{1}, x_{2}\right)$ for arbitrary $p$ and $\alpha$. When $\alpha=0$, we have another zero mode $\rho=\left|x_{1}+i x_{2}\right|^{p} \mathrm{e}^{-\left(x_{1}\right)^{2} / 2-\left(x_{2}\right)^{2} / 2}$ for any $p$. For $p>1$, negative modes appear at small $|\alpha|$. (Note that, when $\alpha=0$, we have an eigenfunction $\rho=\left(x_{1}+i x_{2}\right) \mathrm{e}^{-\left(x_{1}\right)^{2} / 2-\left(x_{2}\right)^{2} / 2}$, which corresponds to the smallest eigenvalue $\lambda=-(p-1)$ for any $p$.) Thus, we can make an argument analogous to the one-variable case (11). Indeed we find for $p=1,2,3$ that the CLM with complexified variables $z_{1}$ and $z_{2}$ gives wrong results at small $|\alpha|$. This can be understood from the behavior of the radial distribution for $r=\left|z_{1}+i z_{2}\right|$.

\section{IMPLICATIONS TO FINITE DENSITY QCD}

Let us discuss the implication of our argument to finite density QCD, which involves the complex fermion determinant $\operatorname{det}(D+m)$ in the partition function, where $D$ represents the Dirac operator and $m$ is the quark mass. The determinant can be written as the product of the eigenvalues $\lambda_{k}$ of $(D+m)$. The drift term of the complex Langevin equation involves $\sum_{k}\left(\lambda_{k}\right)^{-1} \partial \lambda_{k}$, where $\partial$ represents the derivative with respect to the complexified gauge field.

According to our argument, the problem we discussed does not appear as long as the distribution of $\lambda_{k}$ is practically zero at the origin, even if the phase of the fermion determinant rotates frequently during the stochastic process. (See our results for large $p$ in the one-variable case.) This is consistent with the results of recent QCD simulations at finite density, where the distribution of $\lambda_{k}$ has the desired property due either to large quark mass [11] or to high temperature [12]. 
On the other hand, the eigenvalues of $D$ obtained in the CLM are speculated to accumulate at the origin in the chiral limit (corresponding to the $m \rightarrow 0$ limit) when the chiral symmetry is spontaneously broken [13]. If true, the CLM will have problems in that parameter regime unless some new idea is invoked.

\section{SUMMARY}

We have discussed the issue in the CLM concerning the logarithmic singularities in the action. The standard drift term corresponds to regarding the logarithm in the action as a multi-valued function of the complexified variables. The CLM with this drift term is known to give wrong results in some cases. Theoretical understanding of this problem is important, for instance, in applying the method to finite density QCD in the low temperature (confined) phase with light quark mass.

First we emphasized that the multi-valuedness of logarithmic terms in the action cannot be considered the cause of the problem since one can formulate the method without referring to the action, as we have done in section II A. This is also indicated by the example in section IIF.

Rather, the problem should be attributed to the possi- ble breakdown of the key relation between $\rho$ and $P$ due to the singularities in the drift term of the complex Langevin equation. In particular, we pointed out that the relation can be violated due to the boundary terms appearing from integrating by parts in proving (10), which diverges unless $P$ is practically zero around the singularities. This assertion was supported by simple examples. A more quantitative analysis will be reported in the forthcoming publication.

The "FP Hamiltonian" can have negative modes only if the key relation between $\rho$ and $P$ is violated. Note, however, that the key relation can be violated even if the "FP Hamiltonian" does not have negative modes. Hence, the appearance of the negative modes should be regarded merely as an indicator of the violation of the key relation, the latter being the cause of the problem.

To conclude, we hope that the new insights gained in this work will be useful in developing the method further in cases with singularities in the drift term.

\section{ACKNOWLEDGMENTS}

The authors would like to thank D. Sexty for valuable discussions. The work of J. N. was supported in part by Grant-in-Aid for Scientific Research (No. 23244057) from Japan Society for the Promotion of Science.
[1] G. Parisi, Phys. Lett. B 131, 393 (1983).

[2] J. R. Klauder, Phys. Rev. A 29, 2036 (1984).

[3] G. Parisi and Y. s. Wu, Sci. Sin. 24, 483 (1981).

[4] P. H. Damgaard and H. Huffel, Phys. Rept. 152, 227 (1987).

[5] G. Aarts, F. A. James, E. Seiler and I. O. Stamatescu, Eur. Phys. J. C 71, 1756 (2011) arXiv:1101.3270 [heplat]].

[6] E. Seiler, D. Sexty and I. O. Stamatescu, Phys. Lett. B 723, 213 (2013) arXiv:1211.3709 [hep-lat]].

[7] D. Sexty, Phys. Lett. B 729, 108 (2014) arXiv:1307.7748 [hep-lat]].

[8] A. Mollgaard and K. Splittorff, Phys. Rev. D 88, no. 11,
116007 (2013) arXiv:1309.4335 [hep-lat]].

[9] A. Mollgaard and K. Splittorff, Phys. Rev. D 91, no. 3, 036007 (2015) arXiv:1412.2729 [hep-lat]].

[10] J. Greensite, Phys. Rev. D 90, no. 11, 114507 (2014) arXiv:1406.4558 [hep-lat]].

[11] G. Aarts, E. Seiler, D. Sexty and I. O. Stamatescu, Phys. Rev. D 90, no. 11, 114505 (2014) arXiv:1408.3770 [heplat]].

[12] D. Sexty, arXiv:1410.8813 [hep-lat].

[13] K. Splittorff, Phys. Rev. D 91, no. 3, 034507 (2015) arXiv:1412.0502 [hep-lat]]. 